\title{
A bisphosphonate-coating improves the fixation of metal implants in human bone. A randomized trial of dental implants
}

\author{
Jahan Abtahi, Pentti Tengvall and Per Aspenberg
}

\section{Linköping University Post Print}

N.B.: When citing this work, cite the original article.

Original Publication:

Jahan Abtahi, Pentti Tengvall and Per Aspenberg, A bisphosphonate-coating improves the fixation of metal implants in human bone. A randomized trial of dental implants, 2012, Bone, (50), 5, 1148-1151.

http://dx.doi.org/10.1016/j.bone.2012.02.001

Copyright: Elsevier http://www.elsevier.com/

Postprint available at: Linköping University Electronic Press

http://urn.kb.se/resolve?urn=urn:nbn:se:liu:diva-77527 


\section{A bisphosphonate-coating improves the fixation of metal implants in human bone. A randomized trial of dental implants}

Jahan Abtahi DDS, Pentti Tengvall PhD, Per Aspenberg MD, PhD*

Jahan Abtahi

DDS, MD

Department of Oral \& Maxillofacial Surgery, Linköping University Hospital

Department of Experimental and Clinical Medicine,

Faculty of Health Science,

Linkoping University, SE-581 85 Linkoping,

Sweden

E-mail: jahan.abtahi@lio.se

Phone: +46 101031000

Pentti Tengvall

Professor of Biomaterials Science

Department of Biomaterials Science,

Sahlgrenska Academy,

Gothenburg University, SE-405 30, Gothenburg,

Sweden

E-mail: pentti.tengvall@gu.se

Phone: +46 705332608

*Corresponding author:

Per Aspenberg,

Professor of Orthopedics,

Department of Experimental and Clinical Medicine,

Faculty of Health Science,

Linkoping University, SE-581 85 Linkoping,

Sweden

E-mail: per.aspenberg@liu.se

Phone: +46101034166

Mobile phone: +46733866468 


\section{Abstract}

Many surgical procedures use metal implants in bone. The clinical results depend on the strength of the bone holding these implants. Our objective was to show that a drug released from the implant surface can improve parameters reflecting the quality or amount of this bone. Sixteen patients received paired dental titanium implants in the maxilla, in a randomized, double-blinded fashion. One implant in each pair was coated with a thin fibrinogen layer containing 2 bisphosphonates. The other implant was untreated. Fixation was evaluated by measurement of resonance frequency (implant stability quotient; ISQ) serving as a proxy for stiffness of the implant-bone construct. Increase in ISQ at 6 months of follow-up was the primary variable. None of the patients had any complications. The resonance frequency increased 6.9 ISQ units more for the coated implants $(p=0.0001$; Cohen's $d=1.3)$. The average difference in increase in ISQ, and the effect size, suggested a clinically relevant improvement. X-ray showed less bone resorption at the margin of the implant both at 2 months $(p=0.012)$ and at 6 months $(p=0.012)$. In conclusion, a thin, bisphosphonate-eluting fibrinogen coating might improve the fixation of metal implants in human bone. This might lead to new possibilities for orthopedic surgery in osteoporotic bone and for dental implants.

\section{Highlights}

- Dental implants coated with bisphosphonates showed higher resonance frequency.

- Resonance frequency reflects stiffness of the bone-implant construct.

- Randomized double-blinded trial with internal controls.

- First clinical study to show improved implant fixation in bone by coating with a drug.

\section{Key words:}

Bone healing, Dental implant, Drug delivery, Mechanical test, Osseointegration Bisphosphonates. 


\section{Introduction}

1.1 Insertion of metal implants in bone is one of the most common of all surgical procedures. It is part of fracture treatment, joint replacement, and dental surgery. The success of these operations is dependent of the fixation of the implants, which, in turn, depends on the strength of the bone that holds them. If the bone quality is poor, surgical procedures are modified to provide sufficient mechanical fixation by adding more screws or larger devices, or by protecting the implant from mechanical loading for a considerable postoperative time for "osseointegration". Thus, if the quality of the bone holding the implant could be improved locally, surgical procedures would become simpler and rehabilitation would become faster.

1.2 It has been suggested that implants that release growth factors or other drugs that stimulate bone formation would improve implant fixation. This has proven to be successful in animal experiments using a BMP,[1] but so far it has not been shown in the clinic. Also, implants releasing bisphosphonates - a class of drugs that reduce bone resorption-have improved implant fixation in animal experiments.[2] The response to the trauma of implant insertion in cancellous bone involves both bone formation and resorption. These are not entirely coupled, and by reducing resorption, the net amount of bone around the implant will increase. In animal experiments, this leads to fast formation of a shell of new woven bone surrounding the implant, which becomes slowly remodeled into lamellar bone.[3] The shell can become several millimeters thick.[4] Thus, the implant will be held by stronger bone.

1.3 To our knowledge, implants releasing a drug for improvement of fixation have so far not been tested against controlsin humans. Such testing requires a method to measure the quality of fixation, which to our knowledge is available only for dental implants, by measurement of mechanical resonance frequency. The method, its validation and clinical use have been comprehensively reviewed. [5] High-frequency vibrations are applied to the implant, and the frequency at which it will vibrate in resonance is recorded. The resonance vibrations include the bone surrounding the implant, and the stiffer the construct (including the bone), the higher the frequency. Low values predict implant failure, and a change in the value is considered to reflect a change in implant stability.[5]

1.4 Here we report a randomized clinical trial of a drug-releasing implant that was designed for better fixation in bone. We used dental implants, resonance frequency, and a bisphosphonate coating that has been evaluated in a series of animal experiments. $[2,3,6,7]$ The hypothesis was that the coating increases the resonance frequency, reflecting improved fixation.

\section{Patients and methods}

2.1.1 Sixteen patients in need of at least 2 dental implants in the upper jaw at sites with similar bone quality (mean age 65 years, 11 women) received one bisphosphonate-coated implant and 
one ordinary implant. Each patient thus provided his/her own control. All other treatment and follow-up procedures were carried out according to clinical routines. The patients were examined preoperatively by CT-scan to ensure a sufficient maxillary bone volume (in practice a buccoligual distance more than $6 \mathrm{~mm}$ ). Exclusion criteria were: systemic or immunologic disease, drug abuse, uncontrolled diabetes, smoking, previous tumour, trauma or surgery in the maxillary region, or a maxillary bone classified as Cawood and Howell class IV-VI. [8] The patients were recruited between September 2008 and November 2010.

2.1.2 The surgery was performed by the first author at the department of maxillo-facial surgery of Linköping University Hospital. The implants were Brånemark Mk III Ti Unite, 3.75 mm diameter. The coated and control implants were both $11.5 \mathrm{~mm}$ long and visually indistinguishable. Most patients received more than the 2 implants under study; these varied in size between 11.5 and $13 \mathrm{~mm}$. The surgeon chose 2 implantation sites that he considered to be as similar as possible, and named them A and B. A nurse outside the room, who was not otherwise involved in the treatment or in the study, opened a non-transparent randomization envelope. She then delivered either a coated or a control implant for insertion at site A, and the other for site B, depending on the instructions in the envelope. Randomization was performed by the last author by shuffling 16 sealed envelopes in 3 blocks with one crossover between each block, and then marking them with consecutive numbers. After delivering the implants, the nurse placed a paper with the patient's personal identity number and name in the envelope and sealed it again. The envelopes were then stored by the monitor until data lock and unblinding. Thus, the trial was performed double-blind, with exception of the otherwise uninvolved nurse. Implant position is given in Table 1.

2.1.3 After insertion, a resonance frequency analyzer (Osstell Mentor; Integration Diagnostics, Gothenburg, Sweden) was used to measure the implant stability quotient (ISQ) with the transducer oriented perpendicular to the long axis of the implant. The mean of 3 measurements was recorded for each implant.

2.1.4 The implants were then covered with gingiva and left to osseointegrate. After 6 months, the implants were exposed, their resonance frequency was measured, and transgingival abutments for connection with the dental bridge were applied. A few weeks later, the bridge was connected and the patients could start using their new teeth.

2.1.5 Radiographic intraoral films (Insight Super Poly-soft; Kodak, Rochester, NY) were obtained peroperatively and after 2 and 6 months, using a long cone technique. The level of the bone contour at the fixture (marginal bone level) mesially and distally was estimated by the first author by measuring from a reference point on the implant to the first implant-bone contact (Figure 1). Change in bone level over time was expressed as the mean of the changes at the two sides of the implant in $0.25 \mathrm{~mm}$ increments. All radiographic measurements were repeated by an independent, blinded observer. 
2.1.6 The number of patients was predetermined by a power analysis based on a pilot study.[9] All patients signed an informed consent document. The study was registered at clinicaltrials.gov (NCT00767169), was approved by the Regional Ethical Board of Linköping followed the Helsinki declaration of 1983, and was monitored by Linköping Academic Research Center, which reviewed all procedures during the study and checked data before data lock and unblinding. The second and third authors - with possible bias due to economic interests - had no contact with the patients or the data between enrollment of the first patient and data lock.

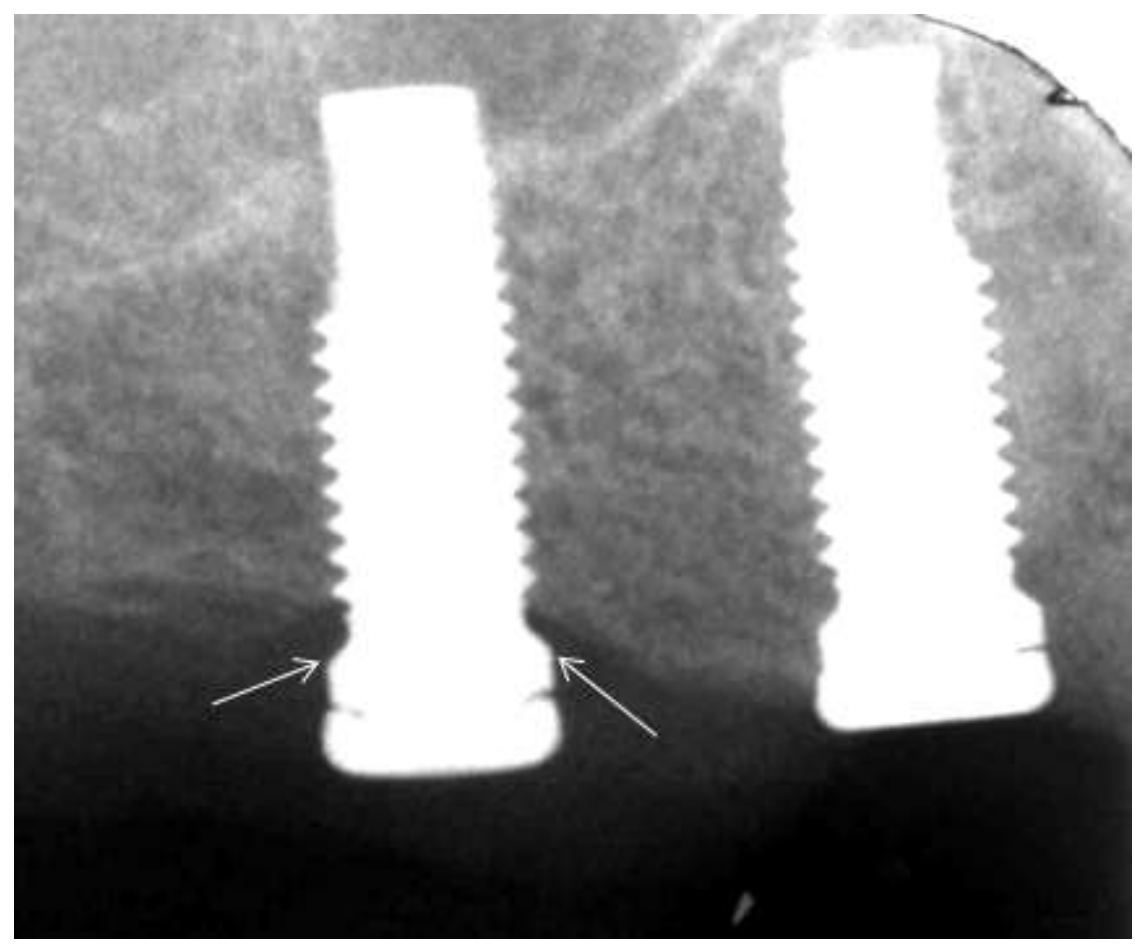

Figure 1. Dental radiograph (patient 16) showing a bisphosphonate coated implant (right) and a control (left). Arrows point at reference points for measurement of marginal bone level.

\section{Implant coating procedure}

2.2.1 The coating procedure was performed as described by Tengvall et al.[2]. Briefly, a crosslinked layer of fibrinogen was covalently bound to the metal, and then small amounts of pamidronate and ibandronate were bound and adsorbed to the fibrinogen matrix.

2.2.2 In detail, the procedure was as follows: The screws were placed in a chamber with $0.2 \mathrm{ml}$ 3-aminopropyltriethoxysilane, $\mathrm{H} 2 \mathrm{~N}(\mathrm{CH} 2) 3 \mathrm{Si}(\mathrm{OC} 2 \mathrm{H} 5) 3$ (APTES; from ABCR, Germany), and baked at $60^{\circ} \mathrm{C}$ at 6 mbar pressure for $10 \mathrm{~min}$, after which the temperature was increased to $150^{\circ} \mathrm{C}$ for $1 \mathrm{~h}$. The surfaces were rinsed for $2 \mathrm{~min}$ in xylene (99\%; Merck) in an ultrasonic bath, rinsed in xylene, and stored in xylene until use - no longer than $1 \mathrm{~h}$. The APTES-coated screws were blown dry with flowing nitrogen and incubated $30 \mathrm{~min}$ in freshly prepared $6 \%$ glutardialdehyde, $\mathrm{HOC}(\mathrm{CH} 2) 3 \mathrm{CHO},(\mathrm{GA})$, in $0.2 \mathrm{M}$ Tris buffer, $\mathrm{pH}$ 9, at room temperature. The surfaces were extensively rinsed and stored in Tris buffer, $\mathrm{pH}$ 9. The fibrinogen matrix was prepared as follows. 
The APTES and glutardialdehyde-coated screws were incubated for $30 \mathrm{~min}$ in $1 \mathrm{mg} / \mathrm{ml}$ protein (human plasminogen-free fibrinogen; Haemochrom Diagnostica, Sweden) dissolved in phosphate-buffered saline (PBS) at $\mathrm{pH}$ 7.4. The surfaces were extensively rinsed in PBS and incubated for $30 \mathrm{~min}$ in a PBS solution at $\mathrm{pH} 5.5$, containing $0.2 \mathrm{M}$ ethyl-dimethylaminopropylcarbodiimide (EDC; Sigma) and 0.05 M N-hydroxy-succinimide (NHS, Sigma). Then a new $1 \mathrm{mg} / \mathrm{ml}$ protein solution was prepared in PBS buffer, $\mathrm{pH}$ 5.5. The surfaces were incubated for $30 \mathrm{~min}$, rinsed in PBS, and again incubated in the EDC/NHS solution. This procedure was repeated 10 times and resulted in an approximately $23-\mathrm{nm}$ thick crosslinked fibrinogen matrix. Two N-bisphosphonates were bound to the fibrinogen-coated surfaces. Pamidronate disodium (Aredia ${ }^{\circledR}, 1 \mathrm{mg} / \mathrm{ml}$ in distilled water; Novartis, Sweden) was first immobilized in the fibrinogen multilayer using the EDC/NHS coupling technique, and finally ibandronate (Bondronate ${ }^{\circledR}, 50 \mu \mathrm{g} / \mathrm{ml}$ in distilled water; Roche, Switzerland) was spontaneously adsorbed during overnight incubation on top of pamidronate-fibrinogen.

2.2.3 The amount of bisphosphonate, approximately $60 \%$ pamidronate and $40 \%$ ibandronate, on similarly treated surfaces has been measured to be less than a microgram per $\mathrm{cm}^{2}$. About $60 \%$ of similarly bound radiolabeled alendronate was released after $8 \mathrm{~h}$ in vitro.

2.2.4 After preparation and sterile packaging, the screws were sterilized by gamma irradiation (25 kGy; ARTIM, Prague, Czech Republic).

\section{Statistics}

2.3.1 Evaluation was based on internal controls. The hypothesis was that bisphosphonate-coated implants would show better fixation. The predetermined primary-effects variable was the increase in ISQ value from insertion to 6 months. This and the absolute ISQ values at 6 months were analyzed by paired t-test. Marginal bone levels on radiographs were analyzed with Wilcoxon's test for paired data.

\section{Results}

3.1 There was no loss to follow-up. There were no surgical complications, and the treatment was clinically successful in all patients. However, 2 control implants had ISQ values less than 57 at 6 months, suggesting insufficient or questionable fixation.

3.2 The bisphosphonate-coated implants showed a larger increase in ISQ value from baseline to 6 months than did the controls (a difference in increase of 6.9 units between experiment and control implants; $95 \%$ confidence interval: 4.1-9.8; $\mathrm{p}=0.0001$ ) (Table 2). All coated implants but 
two showed a higher increase in ISQ value at 6 months than their paired controls. The absolute ISQ value at 6 months was higher for the coated implant in all but one case.

3.3 At 6 months, the marginal bone level was reduced at 9 control screws and 4 bisphosphonate screws. This bone loss was less with bisphosphonate coating than with the controls $(\mathrm{p}=0.012$; Table 3). The difference was already apparent at 2 months $(\mathrm{p}=0.017)$.

3.4 The independent observer also found a significant treatment effect at 6 months $(\mathrm{p}=0.003$; Table 4). The measurements for the bisphosphonate implants at 6 months agreed completely between the two observers for 10 patients, and the difference did not exceed $0.25 \mathrm{~mm}$ among the remaining 6 patients.

\section{Discussion}

4.1 These results represent clinical proof of the principle of improving implant fixation by use of a drug coating. This is different from improvements in implant surface material or texture, which can increase the contact area between implant and bone but probably has less influence on the amount or quality of bone at some distance from the surface. Modifications of dental implants, leading to increased surface energy, have shown a larger increase in ISQ from insertion to 12 weeks than controls in a randomized trial.[10] However, the treatment effect in that trial appeared less than the effect observed in the present trial, suggesting that the use of local drug release might be a more promising principle than physicochemical surface modifications.

4.2 The average improvement in ISQ was clearly larger than 1 standard deviation of the values at any time point, which is conventionally regarded as a large effect size. ISQ values of less than 55 are regarded as indicators of poor fixation, and values below 60 as questionable.[5] The coated implants were all safely above this margin whereas 2 control implants were below this level, and 3 were just above. Even so, this study was not designed to measure clinical benefit as perceived by the patients. The clinical consequences of our findings therefore remain speculative.

4.3 For dental implants, improved fixation would enable surgeons to push the limits regarding the quality of bone (in a surgical sense) in which implants could be inserted. One could possibly also widen the indications for immediate loading. For two-stage procedures, the positive effect on radiographic structure already at 2 months suggests that the time to load bearing could be shortened.

4.4 The coating used in this study represents a "first generation" bisphosphonate coating [2]. While this study was ongoing, improved bisphosphonate coatings have been developed, using zoledronate [11]. Animal experiments have demonstrated that the positive effects are due to the bisphosphonate and not the fibrinogen. Some observations suggest that the fibrinogen alone might have a slightly negative effect on bone implant contact early after insertion, although there is a positive effect when it is combined with a bisphosphonate. [3] [12]. 
4.5 Outside the field of dentistry, one could speculate about bisphosphonate-coated screws for fixation of osteoporotic fractures, improved longevity of external fracture fixation, and better initial stability of joint replacements. It has been shown that bisphosphonate treatment can improve the early stability of total knee replacements.[13 ] For total hip replacement, there is even clinical benefit.[14] However, systemic treatment with bisphosphonates is not without adverse effects.[15] Locally released bisphosphonate from a coated implant will adhere to the nearest bone surface and stay there for a long time, with the bone surface acting as a store for repeated bisphosphonate release.[16] [17] The implants in this study were estimated to carry about a microgram of bisphosphonate, and should have minor systemic effects compared to the weekly systemic mg doses used clinically.

4.6 A potential risk associated with bisphosphonate coated dental implants would be that resorption could be reduced in case of infection, whereby the infected bone could be preserved, leading to chronic osteomyelitis, similar to "osteonecrosis of the jaw" [18]. However, if any such local adverse effects should appear, the problem would be easily solved by removing the bisphosphonate-containing bone in the immediate vicinity of the implant.

\section{Conclusion}

5.1 A thin, bisphosphonate-eluting fibrinogen coating can improve the fixation of metal implants in human bone. This might lead to new possibilities for orthopedic surgery in osteoporotic bone and for dental implants.

\section{Acknowledgements}

The study was funded by the Swedish Research Council (VR 2009-6725).

PA and PT have a patent on the coating procedure, and have shares in a company (Addbio AB) trying to commercialize the principle. PA also has received consulting reimbursement and grants from Eli Lilly \& Co and from Amgen. JA recruited all patients, performed all surgery and all measurements. PT developed and supervised the coating procedure. PA initiated the study, wrote the protocol together with JA and wrote the first manuscript. 
Table 1. Implant position according to ISO 3950.

\begin{tabular}{|rrr|}
\hline Patient & Bisphosphonate & Control \\
\hline $\mathbf{1}$ & 12 & 22 \\
\hline $\mathbf{2}$ & 23 & 13 \\
\hline $\mathbf{3}$ & 24 & 14 \\
\hline $\mathbf{5}$ & 24 & 25 \\
\hline $\mathbf{6}$ & 14 & 24 \\
\hline $\mathbf{7}$ & 21 & 11 \\
\hline $\mathbf{8}$ & 15 & 14 \\
\hline $\mathbf{9}$ & 24 & 14 \\
\hline 10 & 25 & 24 \\
\hline 11 & 11 & 21 \\
\hline 12 & 14 & 24 \\
\hline 13 & 21 & 11 \\
\hline 14 & 14 & 15 \\
\hline 15 & 15 & 14 \\
\hline 16 & 15 & 14 \\
\hline
\end{tabular}


Table 2. Implant stability quotient (ISQ) for paired implants in 16 patients.

\begin{tabular}{|c|c|c|c|c|c|c|c|}
\hline Patient \# & $\begin{array}{l}\text { Control } \\
\text { at } \\
\text { insertion }\end{array}$ & $\begin{array}{l}\text { Control } \\
\text { at } 6 \\
\text { months }\end{array}$ & $\begin{array}{l}\text { Bisphos- } \\
\text { phonate } \\
\text { at } \\
\text { insertion }\end{array}$ & $\begin{array}{l}\text { Bisphos- } \\
\text { phonate } \\
\text { at } 6 \\
\text { months }\end{array}$ & $\begin{array}{l}\text { Increase } \\
\text { for } \\
\text { control }\end{array}$ & $\begin{array}{l}\text { Increase } \\
\text { for } \\
\text { bisphos- } \\
\text { phonate }\end{array}$ & Difference \\
\hline 1 & 57 & 62 & 69 & 85 & 5 & 16 & 11 \\
\hline 2 & 67 & 72 & 70 & 74 & 5 & 4 & -1 \\
\hline 3 & 55 & 70 & 55 & 80 & 15 & 25 & 10 \\
\hline 4 & 73 & 74 & 63 & 75 & 1 & 12 & 11 \\
\hline 5 & 66 & 72 & 50 & 63 & 6 & 13 & 7 \\
\hline 6 & 60 & 64 & 62 & 73 & 4 & 11 & 7 \\
\hline 7 & 57 & 60 & 57 & 68 & 3 & 11 & 8 \\
\hline 8 & 55 & 60 & 57 & 69 & 5 & 12 & 7 \\
\hline 9 & 71 & 69 & 67 & 75 & -2 & 8 & 10 \\
\hline 10 & 69 & 72 & 67 & 78 & 3 & 11 & 8 \\
\hline 11 & 58 & 60 & 65 & 71 & 2 & 6 & 4 \\
\hline 12 & 57 & 68 & 67 & 70 & 11 & 3 & -8 \\
\hline 13 & 59 & 62 & 61 & 70 & 3 & 9 & 6 \\
\hline 14 & 62 & 55 & 59 & 66 & -7 & 7 & 14 \\
\hline 15 & 61 & 64 & 63 & 71 & 3 & 8 & 5 \\
\hline 16 & 62 & 56 & 68 & 74 & -6 & 6 & 12 \\
\hline Mean & 61.8 & 65.0 & 62.5 & 72.6 & 3.2 & 10.1 & 6.9 \\
\hline SD & 5.7 & 6.1 & 5.7 & 5.4 & 5.4 & 5.3 & 5.3 \\
\hline
\end{tabular}


Table 3. Change in marginal bone level from baseline.

\begin{tabular}{rrrrr}
\hline Patient & \multicolumn{2}{c}{ Bisphosphonate } & \multicolumn{2}{c}{ Control } \\
\hline & 2 months & 6 months & 2 months & 6 months \\
\hline $\mathbf{2}$ & -0.25 & -0.25 & 0 & 0 \\
3 & 0 & 0 & 0 & 0 \\
4 & 0 & 0.25 & 0 & 0.25 \\
\hline 5 & 0.25 & 1 & 0,5 & 1 \\
6 & 0 & 0 & 0 & 0 \\
\hline 7 & 0.25 & 0,5 & 1.25 & 0 \\
8 & 0 & 0 & 0 & 0 \\
9 & 0 & 0 & 0 & 0 \\
10 & -0.25 & -0.25 & 1.5 & 2 \\
\hline 11 & 0 & 0 & 0 & 0.25 \\
12 & 0 & 0.25 & 0 & 1 \\
13 & 0 & 0 & 0.25 & 0,5 \\
\hline 14 & 0 & 0 & 0.5 & 1 \\
\hline 15 & 0 & 0 & 0 & 0 \\
\hline 16 & 0 & 0 & 0.75 & 1.25 \\
\hline Max & 0.25 & 1 & 1.5 & 2 \\
\hline Median & 0 & 0 & 0 & 0.25 \\
\hline Min & -0.25 & -0.25 & 0 & 0 \\
\hline
\end{tabular}


Table 4. Change in marginal bone level from baseline; independent observer.

\begin{tabular}{rrrrr}
\hline Patient & \multicolumn{3}{c}{ Bisphosphonate } & \multicolumn{3}{c}{ Control } \\
\hline 1 & 2 months & 6 months & 2 months & 6 months \\
2 & -0.25 & -0.25 & 0 & 0 \\
3 & 0 & 0 & 0 & 0.25 \\
4 & 0 & 0 & 0 & 0.25 \\
5 & 0.25 & 1.5 & 0 & 1.5 \\
6 & 0 & 0 & 0 & 0 \\
7 & 0 & 0 & 0 & 0 \\
8 & 0.5 & 0.5 & 1 & 1.25 \\
9 & 0 & 0 & 0 & 0 \\
10 & 0 & 0 & 0 & 0 \\
11 & 0 & 0 & 1.25 & 1.5 \\
12 & 0 & 0 & 0.25 & 0.25 \\
13 & 0 & 0 & 0 & 1 \\
14 & 0 & 0.25 & 0.25 & 0.5 \\
15 & 0 & 0.25 & 0 & 1.25 \\
\hline 16 & 0 & 0 & 0 & 0.5 \\
\hline Max & 0 & 0 & 1 & 1.25 \\
\hline Median & 0,5 & 1.5 & 1.25 & 1.5 \\
\hline Min & 0 & 0 & 0 & 0.375 \\
\hline
\end{tabular}




\section{References}

[1] Susin C, Qahash M, Polimeni G, Lu PH, Prasad HS, Rohrer MD, Hall J, Wikesjo UM. Alveolar ridge augmentation using implants coated with recombinant human bone morphogenetic protein-7 (rhBMP-7/rhOP-1): histological observations. J Clin Periodontol;37(2010): 574-81.

[2] Tengvall P, Skoglund B, Askendal A, Aspenberg P. Surface immobilized bisphosphonate improves stainless-steel screw fixation in rats. Biomaterials;25(2004): 2133-8.

[3] Wermelin K, Suska F, Tengvall P, Thomsen P, Aspenberg P. Stainless steel screws coated with bisphosphonates gave stronger fixation and more surrounding bone.

Histomorphometry in rats. Bone;42(2008): 365-71.

[4] Bobyn JD, McKenzie K, Karabasz D, Krygier JJ, Tanzer M. Locally delivered bisphosphonate for enhancement of bone formation and implant fixation. J Bone Joint Surg Am;91 Suppl 6(2009): 23-31.

[5] Sennerby L, Meredith N. Implant stability measurements using resonance frequency analysis: biological and biomechanical aspects and clinical implications. Periodontol 2000;47(2008): 51-66.

[6] Wermelin K, Aspenberg P, Linderback P, Tengvall P. Bisphosphonate coating on titanium screws increases mechanical fixation in rat tibia after two weeks. J Biomed Mater Res A;86(2008): 220-7.

[7] Wermelin K, Tengvall P, Aspenberg P. Surface-bound bisphosphonates enhance screw fixation in rats--increasing effect up to 8 weeks after insertion. Acta Orthop;78(2007): 38592.

[8] Cawood JI, Howell RA. A classification of the edentulous jaws. Int J Oral Maxillofac Surg; 17(1988): 232-6.

[9] Abtahi J, Tengvall P, Aspenberg P. Bisphosphonate coating might improve fixation of dental implants in the maxilla: a pilot study. Int J Oral Maxillofac Surg;39673-7.

[10] Schatzle M, Mannchen R, Balbach U, Hammerle CH, Toutenburg H, Jung RE. Stability change of chemically modified sandblasted/acid-etched titanium palatal implants. A randomized-controlled clinical trial. Clin Oral Implants Res;20(2009): 489-95.

[11] Andersson T, Agholme F, Aspenberg P, Tengvall P. Surface immobilized zoledronate improves screw fixation in rat bone: a new method for the coating of metal implants. J Mater Sci Mater Med;213029-37.

[12] Agholme F, Andersson T, Tengvall P, Aspenberg P. Local bisphosphonate release versus hydroxyapatite coating for stainless steel screw fixation in rat tibiae. J Mater Sci Mater Med.

[13] Hilding M, Aspenberg P. Postoperative clodronate decreases prosthetic migration: 4-year follow-up of a randomized radiostereometric study of 50 total knee patients. Acta Orthop;77(2006): 912-6.

[14] Friedl G, Radl R, Stihsen C, Rehak P, Aigner R, Windhager R. The effect of a single infusion of zoledronic acid on early implant migration in total hip arthroplasty. A randomized, double-blind, controlled trial. J Bone Joint Surg Am;91(2009): 274-81.

[15] Schilcher J, Michaelsson K, Aspenberg P. Bisphosphonate use and atypical fractures of the femoral shaft. N Engl J Med;364(2011): 1728-37. 
[16] Russell RG, Watts NB, Ebetino FH, Rogers MJ. Mechanisms of action of bisphosphonates: similarities and differences and their potential influence on clinical efficacy. Osteoporos Int;19(2008): 733-59.

[17] McKenzie K, Dennis Bobyn J, Roberts J, Karabasz D, Tanzer M. Bisphosphonate remains highly localized after elution from porous implants. Clin Orthop Relat Res;469(2011): 51422.

[18] Dodson TB, Raje NS, Caruso PA, Rosenberg AE. Case records of the Massachusetts General Hospital. Case 9-2008. A 65-year-old woman with a nonhealing ulcer of the jaw. N Engl J Med;358(2008): 1283-91. 